\section{Impacto cardiovascular secundario a la terapia para el control del cáncer}

\author{
JUVENAL A. RÍOS ${ }^{1,2,3 a}$, PAMELA ROJO ${ }^{4, b}$, \\ ANDRÉS SCHUSTER ${ }^{5, b}$, JUAN G. GORMAZ ${ }^{6, \mathrm{c}}$, \\ RODRIGO CARRASCO ${ }^{7, \mathrm{a}}$, CAROLINA NEIRA $^{8, \mathrm{~d}, \mathrm{e}}$, \\ SERGIO BECERRA ${ }^{9, \mathrm{~b}, \mathrm{~d}}$, TOMAS P. LABBÉ ${ }^{10, \mathrm{a}}$
}

\section{The cardiovascular impact of cancer control therapy}

Cardiovascular diseases and cancer account for 27 and 25\% of mortality in Chile, respectively. In the last decades, survival of people with cancer has improved due to preventive programs, early detection strategies, advances in technology and development of new antineoplastic therapies. Consequently, a progressive number of cancer-surviving patients have been generated, who may develop cardiovascular diseases, secondary to the same cancer therapy. Cardio-Oncology has emerged as the necessary link between both specialties to promote the prevention and early detection of cardiac complications, in patients undergoing oncological therapies. The aim is to curb cardiovascular complications. Also, to acquire knowledge about the mechanisms and effects of drugs that lead to heart damage aiming to develop efficient cardioprotective therapies. In this article we review and propose a didactic organization and classification of the main cardiovascular effects of cancer control therapy. We recognize that there is still a knowledge gap in basic sciences about the mechanisms that underlie these alterations.

(Rev Med Chile 2020; 148: 93-102)

Key words: Anthracyclines; Cardiotoxicity; Drug Therapy; Neoplasms.
'Departamento de Manejo Integral de Cáncer y otros Tumores, Ministerio de Salud. 2Escuela de Medicina, Facultad de Medicina y Ciencia, Universidad San Sebastián, Lota 2465, Providencia, Santiago, 7510157, Chile.

${ }^{3}$ Programas para el Futuro, Facultad de Estudios Interdisciplinarios y Escuela de Medicina, Facultad de Ciencias, Universidad Mayor.

4Jefe Servicio Cardioimágenes, Unidad de Cardiología, Clínica Dávila.

${ }^{5}$ Departamento de Cardiología, Clínica Alemana de Santiago.

${ }^{6}$ Departamento Oncología, Unidad de Ensayos Clínicos, Clínica Alemana de Santiago.

${ }^{7}$ Servicio de Cardiología, Hospital Salvador. Departamento de Cardiología, Clínica Alemana de Santiago.

${ }^{8}$ Departamento de Enfermedades No Transmisibles, Ministerio de Salud 9 Jefe Departamento de Manejo Integral de Cáncer y otros Tumores, Ministerio de Salud ${ }^{10}$ Unidad de Fisiopatología, Escuela de Medicina, Facultad de Cs. Médicas. Universidad de Santiago de Chile. aM.D., Ph.D.

${ }^{\mathrm{b}} \mathrm{M} . \mathrm{D}$.

'Ph.D.

${ }^{\mathrm{d} M g}$ en Adm. en Salud.

eE.M.

Trabajo no recibió financiamiento. Los autores declaran no tener conflictos de interés.

Recibido el 13 mayo de 2019, aceptado el 30 de agosto de 2019.

Correspondencia a:

Tomás P. Labbé Atenas M.D., Ph.D.

Escuela de Medicina, Facultad de Cs.

Médicas. Universidad de Santiago de Chile.

Santiago, Chile.

labbeatenas@gmail.com

\section{Consideraciones generales}

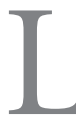

as proyecciones para el año 2050, pronostican que la población de sobrevivientes de un cáncer tendrá un incremento significativo ${ }^{1}$. Esta mayor sobrevida, ha originado un aumento de la prevalencia de enfermedades crónicas, entre ellas las enfermedades cardiovasculares y al mismo tiempo, un incremento en la detección de reacciones adversas secundarias a las terapias oncológicas, en especial aquellas que tienen un impacto a largo plazo (incluso manifestadas clínicamente años más tarde $)^{2,3}$.

Cabe destacar que las enfermedades cardiovasculares y el cáncer, comparten varios factores de riesgo, tales como el sedentarismo, tabaquismo, obesidad y envejecimiento. De hecho, cerca de dos tercios de los pacientes con cáncer, tienen más de 65 años por lo que en ellos se registra un aumento propio de los factores de riesgo cardiovascular; 
lo que contribuye tanto a la aparición como a la progresión de ambas patologías ${ }^{4-6}$.

Está consistentemente reportado que la toxicidad cardiovascular asociada a la terapia oncológica corresponde al principal efecto secundario de la terapia contra el cáncer ${ }^{7}$. La toxicidad cardiovascular puede expresarse clínicamente de diversas formas, destacando la miocardiopatía con disfunción ventricular que puede llevar a insuficiencia cardíaca, infarto agudo al miocardio, miocarditis, enfermedades del pericardio, anormalidades electrofisiológicas, trombosis arterial o venosa e hipertensión arterial o pulmonar ${ }^{8}$.

La Cardio-Oncología ha presentado un marcado desarrollo en los últimos años; lo que se confirma al buscar el número de entradas en la base de datos PUBMED de la palabra "Cardio-oncology" observándose que el número de publicaciones se ha incrementado en los últimos 5 años considerablemente.

A continuación, se organizarán los tipos de daño cardíaco según, tipo de terapia oncológica (quimioterapia, terapia biológica, radioterapia) y algunos síndromes específicos de daño cardíaco. Además, proponemos un esquema simplificado de los tipos de daño por medio un diseño integrado de los principales agentes y efectos biológicos sobre el tejido cardiocirculatorio (Figura 1).

\section{Tipos de daño cardiaco}

\section{Clasificación de daño en función del tipo de terapia oncológica}

Aunque en muchos casos las manifestaciones clínicas y síndromes asociados a toxicidad cardiovascular por terapia oncológica parecieran ser similares, los mecanismos de daño que llevan a dichos desenlaces suelen ser muy variados y están fuertemente relacionados con el tipo de terapia oncológica a la que han sido sometidos los pacientes ${ }^{9,10}$.

\section{Principales mecanismos de daño asociados a disfunción ventricular}

La toxicidad cardiovascular asociada a drogas para el tratamiento del cáncer es el área más importante de la Cardio-Oncología, no solo por representar la principal causa de morbimortalidad cardiaca secundaria a terapia oncológica, sino que también por dar cuenta de la más amplia diversidad de manifestaciones clínicas y mecanismos de daño. Las proyecciones orientan a que seguirá siendo el área de mayor desarrollo dentro la disciplina, dado que el desarrollo de medicamentos para tratar las neoplasias es una de las áreas de mayor crecimiento en la medicina. Dentro de las manifestaciones por drogas oncológicas más estudiadas, se encuentran aquellas que provocan una disfunción ventricular, teniendo como principales exponentes a las antraciclinas y el trastuzumab, cuyos mecanismos de daño exponemos posteriormente.

Diversos estudios de seguimiento a nivel mundial han estimado la incidencia de toxicidad cardiovascular por antraciclínicos, para el cáncer de mama entre 5 y $16 \%$ en función de la dosis acumulada $^{11}$, la cual aumentaría a más de $20 \%$ para la combinación con trastuzumab ${ }^{12}$. Sin embargo, dadas las dificultades para realizar seguimientos de largo plazo y las limitaciones de los métodos de detección, se ha sugerido que las incidencias podrían ser mayores al considerar el universo global de países que se le aplican estas terapias ${ }^{13}$.

\section{Daño tipo I o toxicidad cardiovascular con mecanismo similar a las antraciclinas}

Este tipo de daño es dependiente de dosis y está asociado a cambios estructurales del tejido cardiaco. Se consideraba hasta hace algunos años como un daño mayoritariamente irreversible, sin embargo, desde los hallazgos establecidos por Cardinale el $2015^{14}$, quedó bien establecido que la reversibilidad depende de la precocidad con que se inicia terapia cardiaca específica. Según los conocimientos a la fecha se asociaría a dos mecanismos principales ${ }^{15}$ :

i. Alteración del ciclo Redox: Hipótesis más extendida. La evidencia de esta, señala que inicialmente las antraciclinas tienden a acumularse en las mitocondrias ${ }^{16}$, y debido a sus efectos sobre el complejo I de la cadena transportadora de electrones ${ }^{17}$, se generaría una alta producción de especies reactivas de oxígeno (ROS) nocivas para la integridad del mismo miocardiocito $^{17-20}$.

ii. Inhibición de topoisomerasa $2 \beta$ (top $2 \beta$ ): Este mecanismo de daño sugiere que la interacción de las antraciclinas con la top $2 \beta$ en los miocardiocitos, dificulta la reparación del ADN, evento inicial para el desarrollo de cardiotoxicidad. Esta interacción perpetúa además la disfunción mitocondrial asociada a posterior generación 


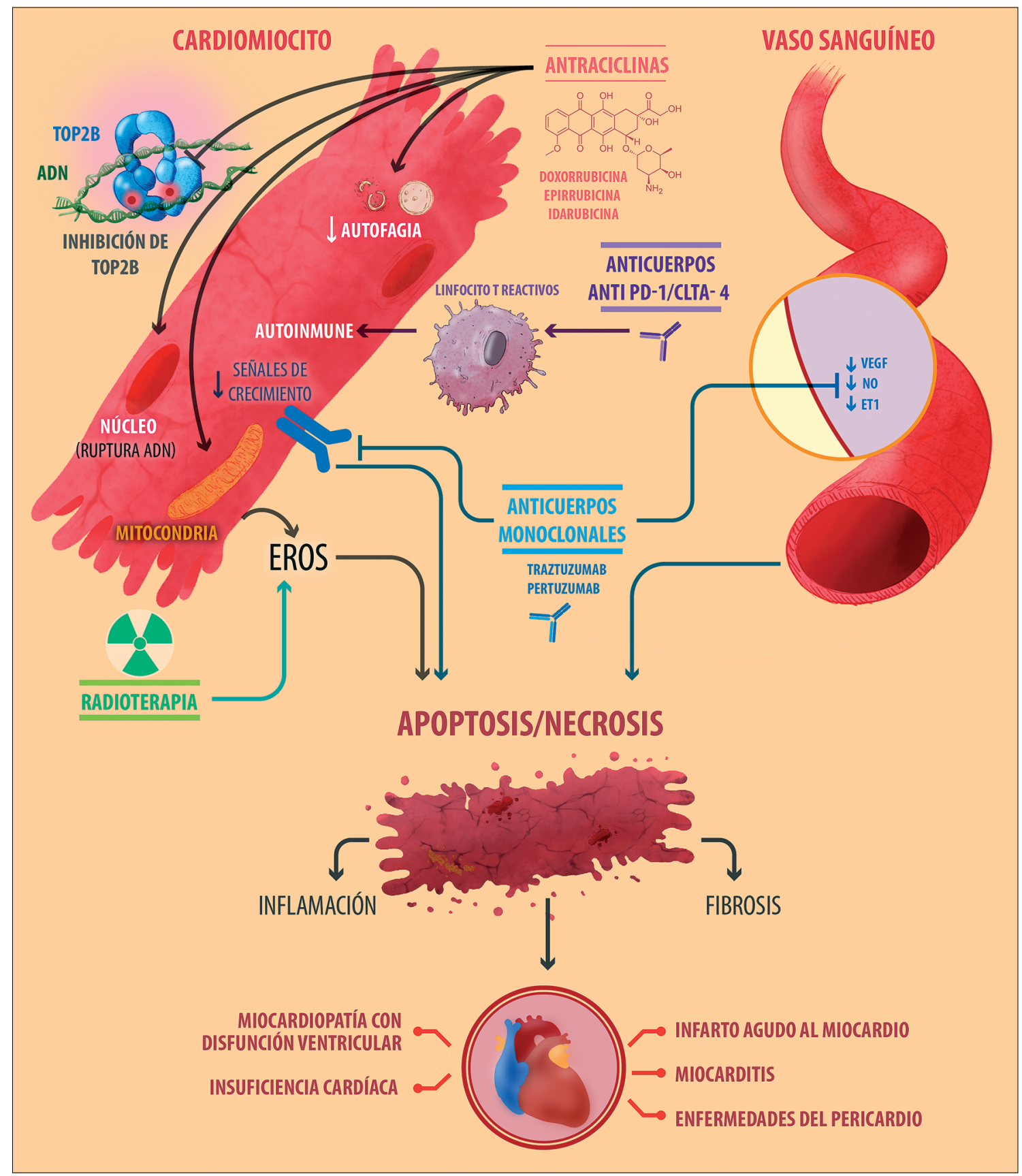

Figura 1. En la figura se muestran los principales mecanismos de daño del cardimiociotio y los vasos sanguíneos. Las antraciclinas generan disminución de la autofagia, inhibición de la actividad de la topll $\beta$, ruptura del ADN, daño mitocondrial y aumento de la producción de especies reactivas del oxígeno (EROS), lo cual es potenciado a su vez por la radioterapia. Asimismo, los anticuerpos monoclonales (traztuzumab y pertuzumab) producen inhibición de las señales de crecimiento (en el cardiomiocito) y disminución de la actividad del factor de crecimiento endotelial (VEGF), óxido nítrico (NO) y endotelina 1 (ET1) en el endotelio vascular. Por su parte la inmunoterapia (anti PD-1/CLTA4) eventualmente podría producir daño autoinmune sobre el miocardio, al activar linfocitos $T$ reactivos que infiltran el parénquima. Todo lo anterior redunda en apoptosis y en algunos casos necrosis, tanto del miocardiocito como del endoteliocito, lo que lleva a daños estructurales y funcionales del tejido cardiovascular propiamente tal, miocardiopatía, insuficiencia cardíaca, infarto agudo al miocardio y miocarditis. 
de ROS (comentado en mecanismo anterior), lo que gatilla, alteración de la homeostasis del calcio, que a su vez fomenta la apoptosis, autofagia e inflamación, procesos que estimulan la fibrosis y remodelación cardíaca ${ }^{21-24}$. Cabe señalar que existen varias formas de topoisomerasas, pero en el caso particular de las células tumorales, éstas expresan niveles elevados de topoisomerasa II alfa (top $2 \alpha$ ), a diferencia de lo que ocurre en los cardiomiocitos que no expresan la top $2 \alpha$, pero expresan una top $2 \beta$. Así, lamentablemente la interacción de las antraciclinas ocurre tanto con la top $2 \alpha$ como con la top $2 \beta$, lo que explicaría el efecto sobre los cardiomiocitos ${ }^{19}$.

iii. Autofagia: Se describe que los antraciclinicos provocarían aumento de la autofagia y mitofagia, mediante la activación de las vías PINK1/Parkin y Bnip3L/Nix las que llevarían finalmente a muerte celular tipo necrosis en los miocardiocitos ${ }^{25}$. Por otro lado, otros autores han visto que doxorrubicina inhibe la autofagia basal en el miocardiocito, mediante el bloqueo de la señalización Akt/mTOR y la disminución de niveles de Beclin 1, en este último caso se postula que la autofagia sería una estrategia celular para contrarrestar la toxicidad cardíaca secundaria a antraciclinas ${ }^{26}$.

iv. Otros mecanismos reportados son el daño directo sobre las células progenitoras cardíacas (CPC), lo que impide aún más la normal regeneración del tejido cardíaco sano y favorece la resolución del daño mediante fibrosis ${ }^{25}$.

\section{Daño tipo II o toxicidad cardiovascular} con mecanismo similar al trastuzumab

La base de este mecanismo es la inhibición de vías que transducen señales al interior de los miocardiocitos, mismas que para las células cancerosas es preciso bloquear. Entre estas vías, destacan, las que controlan el ciclo celular, sobrevida celular y angiogénesis, entre otros. El ejemplo más conocido de este tipo de daño ocurre por bloqueo de la vía de transducción del receptor HER2 a nivel cardiaco, el cual normalmente regula el crecimiento y reparación celular, inhibe la apoptosis y favorece las respuestas al estrés de las células cardiacas. En este caso el trastuzumab, que es un anticuerpo monoclonal dirigido contra este receptor, interrumpe la señalización, afectando la función del cardiomiocito ${ }^{27-30}$.
Además como señaló previamente, la administración de traztuzumab en pacientes que reciben o recibieron quimioterapia con antraciclínicos puede generar una toxicidad sinérgica, principalmente asociada a disfunción ventricular ${ }^{31}$. Este tipo de daño es potencialmente reversible, sin embargo, se ha descrito que un tercio de los casos pueden persistir con disfunción ventricular a largo plazo $^{31}$. Otros anticuerpos monoclonales (pertuzumab, bevacizumab, etc.) e inhibidores de tirosina quinasas (soranefib, sunitinib, imatinib, lapatinib) también son agentes potencialmente cardiotóxicos por este mismo mecanismo ${ }^{32}$.

\section{Daños derivados de radioterapia}

También llamada cardiopatía inducida por radiación (CIR). La incidencia estimada de CIR llega hasta $30 \%$ luego de 10 años de exposición a la radiación y a 54,6\% en seguimiento de 40 años $^{33}$. Se produce principalmente por un daño directo a nivel endotelial, derivado mayoritariamente por la liberación de especies reactivas del oxígeno como respuesta a la radiación ionizante, capaces de inducir inflamación y subsecuente infiltración inflamatoria que deriva en remodelación vascular. La injuria por radioterapia puede afectar el pericardio (provocando daño agudo como derrame pericárdico hasta pericarditis constrictiva en forma crónica), miocardio (condicionando insuficiencia cardiaca), arterias (originando enfermedad coronaria o carotidea), válvulas (causando marcado aumento de calcificación con daño funcional) y sistema excito conductor (desde bloqueos de rama a bloqueos intraventriculares) ${ }^{34,35}$. Puede registrarse un sinergismo del daño miocárdico en pacientes que además reciben quimioterapia (en especial antraciclinas) aunque exista separación temporal entre los tratamientos ${ }^{5,36,37}$.

\section{Toxicidad vascular por terapia antiangiogénica}

La angiogénesis es el proceso de formación de neovasos a partir de vasos prexistentes y es un factor que favorece el crecimiento de tumores y/o metástasis ${ }^{38}$. Uno de los principales exponentes es el factor de crecimiento del endotelio vascular (VEGF) ${ }^{39}$. Los inhibidores de este factor de crecimiento son anticuerpos monoclonales (ej. Bevacizumab) e inhibidores multiquinasa (ej. sorafenib, sunitinib), utilizados para bloquear la angiogénesis tanto de las células tumorales como de las normales. A nivel cardiaco condiciona una 
enfermedad coronaria acelerada, trombosis aguda y vaso espasmo, que podría provocar isquemia miocárdica ${ }^{40}$. A nivel vascular sistémico en tanto, uno de los principales efectos de la inhibición de VEGF es el desarrollo de hipertensión arterial, ya sea como una nueva hipertensión o incrementando las cifras tensionales en pacientes previamente diagnosticados ${ }^{41}$. Los mecanismos involucrados en este caso, se deberían a que la reducción de las vías de señalización del VEGF, provocaría una subsecuente reducción en la producción de vasodilatadores (NO y PGI2), un incremento en la producción de vasoconstrictores (ET-1) y estrés oxidativo, resultando en un incremento del tono vascular y de la remodelación arterial ${ }^{42}$.

\section{Inhibidores del "checkpoints" inmunológicos}

Los inhibidores del "checkpoints" inmunológicos (ICI) representan una novedosa clase de terapia para el cáncer, cuyas bases fueron premiadas el 2018 con el Nobel de Medicina a los profesores Allison y Honjo. La efectividad oncológica de los ICI está dada porque variados tipos de tumores utilizan la sobreexpresión CTLA-4 y PD-1 para escapar de la detección inmune ${ }^{43}$. Sin embargo, su efecto adverso más complejo es la inducción de reacciones autoinmunes a distintos niveles ${ }^{44}$. Es así como esta supresión de reguladores inmunes se ha asociado con dichos eventos adversos inmunológicos hasta en $90 \%$ de los pacientes, incluyendo síntomas cardiológicos, neurológicos, endocrinos, pulmonares, gastrointestinales, renales o dermatológicos ${ }^{45}$.

En el corazón, una expresión fisiológica de CTLA-4 y PD-1 es necesaria para la inhibición de potenciales líneas de células $\mathrm{T}$ autorreactivas a antígenos cardiacos, manteniendo a estos linfocitos en un estado anérgico ${ }^{46}$. Por lo tanto, la cardiotoxicidad de los $\mathrm{ICI}^{47}$ estaría dada mayoritariamente por una pérdida de capacidad de las células cardiacas para generar inmunotolerancia frente a potenciales células T autorreactivas ${ }^{46}$. Específicamente se ha descrito que dichos linfocitos, una vez activados, se expandirían clonalmente para infiltrar corazón, desencadenando una micarditis autoinmune ${ }^{44}$. Esta miocarditis ocurre en aproximadamente en $1 \%$ de los pacientes en tratamiento con ICI y suele ser precoz, presentándose en general durante el primer mes de tratamiento y más frecuentemente con el uso combinado de $\mathrm{ICIs}^{45}$. Por otra parte, de los pacientes que de- sarrollan miocarditis por ICI, en $95 \%$ presenta aumento de troponinas, $90 \%$ tienen alteraciones en ECG, en $65 \%$ de los casos hay elevación del proBNP y cerca de $50 \%$ desarrollan alteraciones de la FEVI. Del punto de vista pronóstico, el grado de elevación de troponinas es un predictor de peor pronóstico y el uso de corticoides en dosis altas en estos pacientes se asocia con una buena respuesta cuando es aplicado en forma temprana ${ }^{45}$.

\section{Clasificación según síndromes clínicos específicos}

Otra forma de clasificar los efectos cardiovasculares de las terapias oncológicas es agrupar las terapias oncológicas según el síndrome clínico que provocan. Es así como entre los principales exponentes de estos cuadros se encuentran:

\section{Enfermedad tromboembólica}

Los pacientes oncológicos presentan la triada de Virchow (lesión endotelial, estasis sanguínea y estado hipercoagulabilidad), cuya base fisiopatológica es la enorme cantidad de citoquinas inflamatorias y factores liberados por la propia masa tumoral, $50 \%$ de los pacientes con cáncer y hasta $90 \%$ con metástasis, presentan alteraciones de los exámenes de coagulación, complejo trombina-antitrombina, fragmento protrombina, fibrinopéptido-A (FPA), PAI-1, factor V Leiden o protrombina. Adicionalmente, ciertos tratamientos (bevacizumab o talidomida) pueden triplicar riesgo de trombosis. Las terapias hormonales (tamoxifeno) o estimulantes de la eritropoyesis (darbepoetina), también aumentan el riesgo de sufrir eventos trombóticos. Se ha estudiado que la expresión de ciertos oncogenes aumentan la hipercoagulabilidad sanguínea y mayor diseminación metastática (en general la mayor activación de "agregación plaquetaria" intratumoral genera trombosis y detiene el sangramiento pero, a su vez, favorece la progresión de las metástasis hematógenas) $)^{48}$.

Los pacientes oncológicos tienen una incidencia 2-20 veces mayor de eventos tromboembólicos que la población sana, especialmente los primeros 3 a 6 meses del diagnóstico, y aumenta si además existe un tratamiento trombogénico. Las neoplasias más asociadas a trombosis asociada a cáncer (TAC) son el cáncer de pulmón, colon y próstata, sin embargo, el riesgo relativo de TAC es 46 veces mayor en paciente con mieloma múltiple, 20 veces 
mayor en cáncer cerebral y 16 veces mayor en cáncer de páncreas. En fase terminal o metastásica, los cánceres de estómago, vejiga, útero, riñón y pulmón también se asocian a alta incidencia de trombosis venosa $(\mathrm{TV})^{49}$.

Los factores predisponentes más significativos para la TAC, han sido la cirugía, quimioterapia y radioterapia, hospitalizaciones prolongadas y postración ${ }^{50}$. Otros predictores de mayor mortalidad son la progresión de la enfermedad (aparición de metástasis), tipo de cáncer, recurrencia de eventos trombóticos venosos (hasta en 7\% de los casos), hemorragia mayor (8\%) e insuficiencia renal ${ }^{51}$.

\section{Isquemia miocárdica}

Se ha descrito que diversos tratamientos antineoplásicos aumentan el riesgo de enfermedad coronaria. Se ha reportado que capecitabina, 5-fluoruracilo, paclitaxel, docetaxel, bevacizumab, sorafenib, nilotinib, ponatinib, agonistas de la hormona liberadora de gonadotrofina (GnRH) y el ácido retinoico están asociados a un mayor riesgo isquemia miocárdica por distintos mecanismos. Por otra parte, la radioterapia supra diafragmática, al tener efectos directos de remodelación sobre el endotelio vascular coronario, también favorecería la incidencia de síndromes coronarios $^{52}$.

\section{Alteraciones electrofisiológicas}

Los trastornos del ritmo derivados del tratamiento antineoplásico obedecen en general al bloqueo de canales de potasio, entradas masivas de calcio, apoptosis y fragmentación del ADN en las células del sistema exitoconductor lo que lleva a disfunción del sistema His-Purkinje. Además, en forma indirecta se puede observar eventos arrítmicos secundarios a isquemia por espasmo coronario relacionado a terapias oncológicas ${ }^{53,54,55}$.

\section{Enfermedad del pericardio}

Habitualmente relacionada con la radioterapia. Se presenta en fase aguda como derrame pericárdico o pericarditis. La constricción pericárdica habitualmente es una expresión tardía, lo que obliga el control periódico y a largo plazo de los pacientes sometidos a esta terapia, aconsejándose estudios cada 5 años $^{56,57}$.

\section{Hipertensión arterial}

Como se señaló previamente, se ha descrito principalmente en relación con el uso de anti- cuerpos anti-VEGF, por lo que se recomienda monitorización estricta de presión arterial en estos pacientes. Sin embargo, existen otras terapias oncológicas tales como agentes alquilantes, esteroides y eritropoyetina que también están asociados a un incremento de la presión arterial ${ }^{58}$.

\section{Enfermedad arterial periférica}

Diversos fármacos antineoplásicos, tanto de quimioterapia como específicas se han descrito como inductores de daño microvascular periférico. Estos al gatillar un incremento de la remodelación vascular, podrían desencadenar una ateroesclerosis acelerada, determinando claudicación de las extremidades, si se asocia a fenómenos de trombosis arterial, podría incrementar el riesgo de eventos de isquemia crítica en extremidades. Se ha atribuido este efecto adverso al cisplatino, 5-fluoruracilo, gemcitabina, inhibidores de aromatasa, talidomida, bevacizumab, sunitinib y sorafenib ${ }^{59}$.

\section{Conclusiones y desafíos}

Históricamente, la mayoría de los estudios que han reportado toxicidad cardiovascular se han enfocado en los efectos del uso de las antraciclinas y quimioterapéuticos clásicos. Sin embargo, como vimos en esta revisión, las nuevas terapias dirigidas (inhibidores de tirosina quinasa y anticuerpos monoclonales) también se deben agregar al listado de terapias que pueden provocar toxicidad cardiovascular.

Existe un amplio consenso en la comunidad médica internacional que la disfunción ventricular y/o insuficiencia cardiaca, es una de las principales manifestaciones de toxicidad cardiovascular por tratamientos oncológicos, sin embargo, como vimos en esta revisión no es la única forma. En esta misma línea, queda de manifiesto que aún existe un vacío importante en los detalles celulares y moleculares que explican cada uno de los efectos adversos de la terapia oncológica en el músculo cardíaco, las estructuras cardíacas anexas (válvulas, pericardio) y los propios vasos sanguíneos, lo que explicaría en gran parte por qué la batería de drogas específicas para frenar este especial tipo de daño, en la actualidad, es muy limitada y no han habido grandes desarrollos farmacológicos.

En otra línea, debemos considerar que los 
costos del tratamiento cardiológico pueden superar ampliamente a los asociados a la terapia oncológica exitosa en cánceres de buen pronóstico y que son pesquisados tempranamente, esto asociado al hecho de que existe una significativa perdida de años de vida saludable en la población de sobrevivientes de cáncer, por aumento de invalidez secundaria, entre otras razones, también a la insuficiencia cardíaca con el consecuente impacto a nivel laboral y social.

El desarrollo y fomento de los equipos "Cardio-Oncología" ha sido relevado por otros autores chilenos previamente ${ }^{60}$, instalándose como idea primordial la cohabitación en una misma unidad clínica de los oncólogos médicos, radioterapeutas, cardiólogos, enfermeras especializadas, investigadores y otros profesionales relacionados al tratamiento del cáncer. Por último y a pesar de la relevancia que ha tenido el Plan Nacional de Cáncer en Chile y considerando el advenimiento de la prevención cuaternaria como una forma de hacer medicina, debemos mantener en el horizonte que curar a una población de una enfermedad crónica no debe ser motivo para instalarle otra enfermedad crónica, muchas veces igual o más compleja y con costos similares para el Estado, "no intercambiemos enfermedad, por enfermedad" decimos.

Agradecimientos: Agradecemos especialmente al renovado equipo del Departamento de Manejo Integral de Cáncer y otros Tumores, del Ministerio de Salud de Chile; Fanny Sepúlveda, Gina Merino, Priscilla González, Dunja Roje, Carolina Zárate, Marlene Valenzuela y al Dr. Tomás Merino. Por su labor incansable al servicio público y por otorgarnos el tiempo para escribir literatura científica, en paralelo a las contingencias que día a día, conlleva el trabajo propio de la cartera de salud. Agradecemos también, a la Dra. Melanie Paccot, Jefa del Departamento de Enfermedades No Transmisibles (ENT) del Ministerio de Salud, por apoyar desde su departamento, el desarrollo de ésta importante línea de trabajo conjunta, entre Cáncer y ENT, como es el "Manejo Cardiovascular del Paciente con Cáncer”.

\section{Referencias}

1. Curigliano G, Cardinale D, Dent S, Criscitiello C, Aseyev O, Lenihan D, et al. Cardiotoxicity of anticancer treatments: Epidemiology, detection, and management.
CA Cancer J Clin [Internet]. 2016 Jul 1 [cited 2018 Dec 10];66(4):309-25. Available from: http://doi.wiley. com/10.3322/caac. 21341

2. Kalam K, Marwick TH. Role of cardioprotective therapy for prevention of cardiotoxicity with chemotherapy: A systematic review and meta-analysis. Eur J Cancer [Internet]. 2013 Sep [cited 2018 Dec 18];49(13):29009. Available from: http://www.ncbi.nlm.nih.gov/pubmed/23706982

3. Miller KD, Siegel RL, Lin CC, Mariotto AB, Kramer JL, Rowland JH, et al. Cancer treatment and survivorship statistics, 2016. CA Cancer J Clin [Internet]. 2016 Jul 1 [cited 2018 Dec 10];66(4):271-89. Available from: http:// doi.wiley.com/10.3322/caac.21349

4. Johnson CB, Davis MK, Law A, Sulpher J. Shared Risk Factors for Cardiovascular Disease and Cancer: Implications for Preventive Health and Clinical Care in Oncology Patients. Can J Cardiol [Internet]. 2016 Jul 1 [cited 2018 Dec 10];32(7):900-7. Available from: https://www.sciencedirect.com/science/article/pii/ S0828282X16300526?via\%3Dihub

5. Aleman BMP, Moser EC, Nuver J, Suter TM, Maraldo MV, Specht L, et al. Cardiovascular disease after cancer therapy. Eur J Cancer Suppl [Internet]. 2014 Jun 1 [cited 2018 Dec 10];12(1):18-28. Available from: https://www.sciencedirect.com/science/article/pii/ S1359634914000044

6. Al-Kindi SG, Oliveira GH. Prevalence of Preexisting Cardiovascular Disease in Patients With Different Types of Cancer: The Unmet Need for Onco-Cardiology. Mayo Clin Proc [Internet]. 2016 Jan 1 [cited 2018 Dec 10];91(1):81-3. Available from: https://www.sciencedirect.com/science/article/abs/pii/S0025619615007387?via\%3Dihub

7. Bowles EJA, Wellman R, Feigelson HS, Onitilo AA, Freedman AN, Delate T, et al. Risk of Heart Failure in Breast Cancer Patients After Anthracycline and Trastuzumab Treatment: A Retrospective Cohort Study. JNCI J Natl Cancer Inst [Internet]. 2012 Sep 5 [cited 2018 Dec 10];104(17):1293-305. Available from: https:// academic.oup.com/jnci/article-lookup/doi/10.1093/jnci/ djs317

8. Savarese G, Lund LH. Global Public Health Burden of Heart Failure. Card Fail Rev [Internet]. 2017 Apr [cited 2018 Dec 10];3(1):7-11. Available from: http://www. ncbi.nlm.nih.gov/pubmed/28785469

9. Moslehi JJ. Cardiovascular Toxic Effects of Targeted Cancer Therapies. Longo DL, editor. N Engl J Med [Internet]. 2016 Oct 13 [cited 2019 Aug 16];375(15):145767. Available from: http://www.nejm.org/doi/10.1056/ NEJMra1 100265 
10. Kitsis RN, Riquelme JA, Lavandero S. Heart Disease and Cancer. Circulation [Internet]. 2018 Aug 14 [cited 2019 Aug 16];138(7):692-5. Available from: http://www.ncbi. nlm.nih.gov/pubmed/30359137

11. Vejpongsa P, Yeh ETH. Prevention of Anthracycline-Induced Cardiotoxicity: Challenges and Opportunities. J Am Coll Cardiol [Internet]. 2014 Sep 2 [cited 2018 Dec 10];64(9):938-45. Available from: https://www.sciencedirect.com/science/article/pii/S0735109714043162?via $\% 3$ Dihub

12. Bowles EJA, Wellman R, Feigelson HS, Onitilo AA, Freedman AN, Delate T, et al. Risk of heart failure in breast cancer patients after anthracycline and trastuzumab treatment: a retrospective cohort study. J Natl Cancer Inst [Internet]. 2012 Sep 5 [cited 2019 Aug 16];104(17):1293-305. Available from: https://academic. oup.com/jnci/article-lookup/doi/10.1093/jnci/djs317

13. Guglin M, Munster P, Fink A, Krischer J. Lisinopril or Coreg $\mathrm{CR}$ in reducing cardiotoxicity in women with breast cancer receiving trastuzumab: A rationale and design of a randomized clinical trial. Am Heart J [Internet]. 2017 Jun [cited 2019 Aug 16];188:87-92. Available from: https://linkinghub.elsevier.com/retrieve/ pii/S0002870317300935

14. Cardinale D, Colombo A, Bacchiani G, Tedeschi I, Meroni CA, Veglia F, et al. Early detection of anthracycline cardiotoxicity and improvement with heart failure therapy. Circulation 2015; 131 (22): 1981-8.

15. Ewer MS, Lenihan DJ. Left ventricular ejection fraction and cardiotoxicity: is our ear really to the ground? J Clin Oncol [Internet]. 2008 Mar 10 [cited 2018 Dec 11]; 26 (8): 1201-3. Available from: http://ascopubs.org/ doi/10.1200/JCO.2007.14.8742

16. Anderson AB, Xiong G, Arriaga EA. Doxorubicin Accumulation in Individually Electrophoresed Organelles. 2004 [cited 2018 Dec 11]; Available from: https://pubs. acs.org/doi/abs/10.1021/ja0492539

17. Lebrecht D, Kokkori A, Ketelsen U-P, Setzer B, Walker UA. Tissue-specific mtDNA lesions and radical-associated mitochondrial dysfunction in human hearts exposed to doxorubicin. J Pathol [Internet]. 2005 Dec 1 [cited 2018 Dec 11]; 207 (4): 436-44. Available from: http:// doi.wiley.com/10.1002/path.1863

18. Šimùnek $T$, Štìrba $M$, Popelová $O$, Adamcová $M, H r-$ dina R, Geršl V. Anthracycline-induced cardiotoxicity: Overview of studies examining the roles of oxidative stress and free cellular iron [Internet]. 2009 [cited 2018 Dec 11]. Available from: http://www.if-pan.krakow.pl/ pjp/pdf/2009/1_154.pdf

19. Zhang S, Liu X, Bawa-Khalfe T, Lu L-S, Lyu YL, Liu LF, et al. Identification of the molecular basis of doxorubi- cin-induced cardiotoxicity. Nat Med [Internet]. 2012 Nov 28 [cited 2018 Dec 11]; 18 (11): 1639-42. Available from: http://www.nature.com/articles/nm.2919

20. Lencová-Popelová O, Jirkovský E, Mazurová Y, Lenco J, Adamcová M, Šimunek T, et al. Molecular Remodeling of Left and Right Ventricular Myocardium in Chronic Anthracycline Cardiotoxicity and Post-Treatment Follow Up. Calvert J, editor. PLoS One [Internet]. 2014 May 7 [cited 2018 Dec 11]; 9 (5): e96055. Available from: https://dx.plos.org/10.1371/journal.pone.0096055

21. Wang JC. Cellular roles of DNA topoisomerases: a molecular perspective. Nat Rev Mol Cell Biol [Internet]. 2002 Jun [cited 2018 Dec 13]; 3 (6): 430-40. Available from: http://www.ncbi.nlm.nih.gov/pubmed/12042765

22. Ghigo A, Li M, Hirsch E. New signal transduction paradigms in anthracycline-induced cardiotoxicity. Biochim Biophys Acta - Mol Cell Res [Internet]. 2016 Jul [cited 2018 Dec 13]; 1863 (7): 1916-25. Available from: http:// www.ncbi.nlm.nih.gov/pubmed/26828775

23. Bodley A, Liu LF, Israel M, Seshadri R, Koseki Y, Giuliani FC, et al. DNA topoisomerase II-mediated interaction of doxorubicin and daunorubicin congeners with DNA. Cancer Res [Internet]. 1989 Nov 1 [cited 2018 Dec 13]; 49 (21): 5969-78. Available from: http://www.ncbi.nlm. nih.gov/pubmed/2551497

24. Lyu YL, Kerrigan JE, Lin C-P, Azarova AM, Tsai Y-C, Ban Y, et al. Topoisomerase II -Mediated DNA Double-Strand Breaks: Implications in Doxorubicin Cardiotoxicity and Prevention by Dexrazoxane. Cancer Res [Internet]. 2007 Sep 15 [cited 2018 Dec 13]; 67 (18): 8839-46. Available from: http://www.ncbi.nlm.nih.gov/ pubmed/17875725

25. Nebigil CG, Désaubry L. Updates in Anthracycline-Mediated Cardiotoxicity. Front Pharmacol [Internet]. 2018 Nov 12 [cited 2019 Aug 16]; 9: 1262. Available from: http://www.ncbi.nlm.nih.gov/pubmed/30483123

26. Pizarro M, Troncoso R, Martínez GJ, Chiong M, Castro $\mathrm{PF}$, Lavandero S. Basal autophagy protects cardiomyocytes from doxorubicin-induced toxicity. Toxicology [Internet]. 2016 Aug 31 [cited 2019 Aug 16]; 370: 41-8. Available from: http://www.ncbi.nlm.nih.gov/pubmed/27666003

27. Crone SA, Zhao Y-Y, Fan L, Gu Y, Minamisawa S, Liu $\mathrm{Y}$, et al. ErbB2 is essential in the prevention of dilated cardiomyopathy. Nat Med [Internet]. 2002 May 1 [cited 2018 Dec 13]; 8 (5): 459-65. Available from: http://www. nature.com/articles/nm0502-459

28. Krause DS, Van Etten RA. Tyrosine Kinases as Targets for Cancer Therapy. N Engl J Med [Internet]. 2005 Jul 14 [cited 2018 Dec 13]; 353 (2): 172-87. Available from: http://www.nejm.org/doi/abs/10.1056/NEJMra044389 
29. Guglin M, Cutro R, Mishkin JD. Trastuzumab-Induced Cardiomyopathy. J Card Fail [Internet]. 2008 Jun 1 [cited 2018 Dec 13]; 14 (5): 437-44. Available from: https://www.sciencedirect.com/science/article/pii/ S1071916408000651?via\%3Dihub

30. Markman TM, Markman M. Cardio-Oncology: mechanisms of cardiovascular toxicity. F1000Research [Internet]. 2018 Jan 25 [cited 2018 Dec 13]; 7: 113. Available from: https://f1000research.com/articles/7-113/v1

31. Darby SC, McGale P, Taylor CW, Peto R. Long-term mortality from heart disease and lung cancer after radiotherapy for early breast cancer: prospective cohort study of about 300,000 women in US SEER cancer registries. Lancet Oncol [Internet]. 2005 Aug 1 [cited 2018 Dec 13]; 6 (8): 557-65. Available from: https://www.sciencedirect.com/science/article/pii/S1470204505702515?via\%3Dihub

32. Babiker HM, McBride A, Newton M, Boehmer LM, Drucker AG, Gowan M, et al. Cardiotoxic effects of chemotherapy: A review of both cytotoxic and molecular targeted oncology therapies and their effect on the cardiovascular system. Crit Rev Oncol Hematol [Internet]. 2018 Jun 1 [cited 2018 Dec 13]; 126: 186-200. Available from: https:/www.sciencedirect.com/science/article/pii/ S1040842817303566?via\%3Dihub

33. Carver JR, Shapiro CL, Ng A, Jacobs L, Schwartz C, Virgo KS, et al. American Society of Clinical Oncology clinical evidence review on the ongoing care of adult cancer survivors: cardiac and pulmonary late effects. J Clin Oncol [Internet]. 2007 Sep 1 [cited 2018 Dec 13]; 25 (25): 3991-4008. Available from: http://ascopubs.org/ doi/10.1200/JCO.2007.10.9777

34. Heidenreich PA, Kapoor JR. Radiation induced heart disease. Heart [Internet]. 2008 Nov 25 [cited 2018 Dec 13];95(3):252-8. Available from: http://www.ncbi.nlm. nih.gov/pubmed/19144884

35. Hooning MJ, Botma A, Aleman BMP, Baaijens MHA, Bartelink H, Klijn JGM, et al. Long-Term Risk of Cardiovascular Disease in 10-Year Survivors of Breast Cancer. JNCI J Natl Cancer Inst [Internet]. 2007 Mar 7 [cited 2018 Dec 13]; 99 (5): 365-75. Available from: https:// academic.oup.com/jnci/article-lookup/doi/10.1093/jnci/ djk064

36. Aleman BMP, van den Belt-Dusebout AW, De Bruin ML, van 't Veer MB, Baaijens MHA, Boer JP, et al. Late cardiotoxicity after treatment for Hodgkin lymphoma. Blood [Internet]. 2007 Mar 1 [cited 2018 Dec 13]; 109 (5): 1878-86. Available from: http://www.ncbi.nlm.nih. gov/pubmed/17119114

37. Darby SC, Cutter DJ, Boerma M, Constine LS, Fajardo LF, Kodama K, et al. Radiation-related heart disease: current knowledge and future prospects. Int J Radiat Oncol Biol Phys [Internet]. 2010 Mar 1 [cited 2018 Dec 13]; 76 (3): 656-65. Available from: http://www.ncbi. nlm.nih.gov/pubmed/20159360

38. Ferrara N, Gerber H-P, LeCouter J. The biology of VEGF and its receptors. Nat Med [Internet]. 2003 Jun 1 [cited 2018 Dec 13]; 9 (6): 669-76. Available from: http://www.nature.com/articles/nm0603-669

39. Lankhorst S, Saleh L, Danser AJ, van den Meiracker AH. Etiology of angiogenesis inhibition-related hypertension. Curr Opin Pharmacol [Internet]. 2015 Apr 1 [cited 2018 Dec 13]; 21: 7-13. Available from: https://www.sciencedirect.com/science/article/pii/ S1471489214001659?via\%3Dihub

40. Cortés J, Calvo V, Ramírez-Merino N, O’Shaughnessy J, Brufsky A, Robert N, et al. Adverse events risk associated with bevacizumab addition to breast cancer chemotherapy: a meta-analysis. Ann Oncol [Internet]. 2012 May 1 [cited 2018 Dec 13]; 23 (5): 1130-7. Available from: http://www.ncbi.nlm.nih.gov/ pubmed/21976387

41. Milán A, Puglisi E, Ferrari L, Bruno G, Losano I, Veglio F. Arterial hypertension and cancer. Int J Cancer [Internet]. 2014 May 15 [cited 2019 Aug 16]; 134 (10): 2269-77. Available from: http://www.ncbi.nlm.nih.gov/ pubmed/23784914

42. Touyz RM, Herrmann SMS, Herrmann J. Vascular toxicities with VEGF inhibitor therapies-focus on hypertension and arterial thrombotic events. J Am Soc Hypertens [Internet]. 2018 Jun [cited 2019 Aug 16];12(6):409-25. Available from: http://www.ncbi.nlm. nih.gov/pubmed/29703600

43. Darvin P, Toor SM, Sasidharan Nair V, Elkord E. Immune checkpoint inhibitors: recent progress and potential biomarkers. Exp Mol Med [Internet]. 2018 Dec 13 [cited 2019 Aug 16]; 50 (12): 165. Available from: http:// www.ncbi.nlm.nih.gov/pubmed/30546008

44. Postow MA, Sidlow R, Hellmann MD. Immune-Related Adverse Events Associated with Immune Checkpoint Blockade. Longo DL, editor. N Engl J Med [Internet]. 2018 Jan 11 [cited 2019 Aug 16]; 378 (2): 158-68. Available from: http://www.nejm.org/doi/10.1056/ NEJMra1703481

45. Mahmood SS, Fradley MG, Cohen JV, Nohria A, Reynolds KL, Heinzerling LM, et al. Myocarditis in Patients Treated With Immune Checkpoint Inhibitors. J Am Coll Cardiol [Internet]. 2018 Apr 24 [cited 2019 Aug 16]; 71 (16): 1755-64. Available from: http://www.ncbi.nlm.nih. gov/pubmed/29567210

46. Tajiri K, Ieda M. Cardiac Complications in Immune Checkpoint Inhibition Therapy. Front Cardiovasc Med 
[Internet]. 2019 [cited 2019 Aug 16]; 6: 3. Available from: http://www.ncbi.nlm.nih.gov/pubmed/30729114

47. Pradhan R, Nautiyal A, Singh S. Diagnosis of immune checkpoint inhibitor-associated myocarditis: A systematic review. Int J Cardiol [Internet]. 2019 Jul 11 [cited 2019 Aug 16]; Available from: http://www.ncbi.nlm.nih. gov/pubmed/31327516

48. Falanga A, Russo L, Milesi V, Vignoli A. Mechanisms and risk factors of thrombosis in cancer. Crit Rev Oncol Hematol [Internet]. 2017 Oct [cited 2019 Aug 16]; 118: 79-83. Available from: http://www.ncbi.nlm.nih.gov/ pubmed/28917273

49. Imberti D, Agnelli G, Ageno W, Moia M, Palareti G, Pistelli R, et al. Clinical characteristics and management of cancer-associated acute venous thromboembolism: findings from the MASTER Registry. Haematologica [Internet]. 2008 Feb 1 [cited 2019 Aug 16]; 93 (2): 273-8. Available from: http://www.ncbi.nlm.nih.gov/ pubmed/18223291

50. Jara-Palomares L, Otero R, Jiménez D, Carrier M, Tzoran I, Brenner B, et al. Development of a Risk Prediction Score for Occult Cancer in Patients With VTE. Chest [Internet]. 2017 Mar [cited 2019 Aug 16]; 151 (3): 564-71. Available from: http://www.ncbi.nlm.nih.gov/ pubmed/27815153

51. Ay C, Dunkler D, Marosi C, Chiriac A-L, Vormittag R, Simanek R, et al. Prediction of venous thromboembolism in cancer patients. Blood [Internet]. 2010 Dec 9 [cited 2019 Aug 16]; 116 (24): 5377-82. Available from: http://www.ncbi.nlm.nih.gov/pubmed/20829374

52. Eschenhagen T, Force T, Ewer MS, de Keulenaer GW, Suter TM, Anker SD, et al. Cardiovascular side effects of cancer therapies: a position statement from the Heart Failure Association of the European Society of Cardiology. Eur J Heart Fail [Internet]. 2011 Jan 1 [cited 2018 Dec 10]; 13 (1): 1-10. Available from: http://doi.wiley. com/10.1093/eurjhf/hfq213

53. Kilickap S, Barista I, Akgul E, Aytemir K, Aksoy S, Tekuzman G. Early and Late Arrhythmogenic Effects of Doxorubicin. South Med J [Internet]. 2007 Mar [cited 2018 Dec 18]; 100 (3): 262-5. Available from: http://sma. org/southern-medical-journal/article/early-and-late-arrhythmogenic-effects-of-doxorubicin

54. Guglin M, Aljayeh M, Saiyad S, Ali R, Curtis AB. Intro- ducing a new entity: chemotherapy-induced arrhythmia. Europace [Internet]. 2009 Dec 1 [cited 2018 Dec 18]; 11 (12): 1579-86. Available from: https://academic.oup. com/europace/article-lookup/doi/10.1093/europace/ eup300

55. Tarantini L, Massimo Gulizia M, Di Lenarda A, Maurea $\mathrm{N}$, Giuseppe Abrignani M, Bisceglia I, et al. ANMCO/ AIOM/AICO Consensus Document on clinical and management pathways of cardio-oncology: executive summary Consensus Document Approval Faculty in Appendix. Eur Hear J Suppl [Internet]. 2017 [cited 2018 Dec 18]; 19. Available from: https://pdfs.semanticscholar.org/b67f/dc203c0d7e9343888bb453f978bf6b88e3ed. pdf

56. Zamorano JL, Lancellotti P, Rodríguez Muñoz D, Aboyans V, Asteggiano R, Galderisi M, et al. 2016 ESC Position Paper on cancer treatments and cardiovascular toxicity developed under the auspices of the ESC Committee for Practice Guidelines. Eur Heart J [Internet]. 2016 Sep 21 [cited 2018 Dec 18]; 37 (36): 2768-801. Available from: https://academic.oup.com/eurheartj/ article-lookup/doi/10.1093/eurheartj/ehw211

57. Senkus-Konefka E, Jassem J. Cardiovascular effects of breast cancer radiotherapy. Cancer Treat Rev [Internet]. 2007 Oct 1 [cited 2018 Dec 18]; 33 (6): 578-93. Available from: https://www.sciencedirect.com/science/article/pii/ S0305737207000801?via\%3Dihub

58. Spanish Society Of Cardiology. Cardio-oncology : theory book + case study book. [Internet]. Cto Editorial; 2015 [cited 2018 Dec 13]. Available from: https://secardiologia.es/publicaciones/catalogo/libros/6506-cardio-oncology

59. Davignon J. Role of Endothelial Dysfunction in Atherosclerosis. Circulation [Internet]. 2004 Jun 15 [cited 2018 Dec 13]; 109 (23_suppl_1): III-27-III-32. Available from: http://circ.ahajournals.org/cgi/doi/10.1161/01. CIR.0000131515.03336.f8

60. Hameau R, Gabrielli L, Garrido M, Guzmán AM, Retamal I, Vacarezza MJ, et al. Cardiotoxicidad inducida por tratamientos oncológicos. Fundamentos para la implementación de equipos de Cardio-Oncología [Internet]. Vol. 146, Rev Med Chile 2018 [cited 2018 Dec 18]. Available from: https://scielo.conicyt.cl/pdf/rmc/ v146n1/0034-9887-rmc-146-01-0068.pdf 